Research Article

\title{
Automatic Decision Algorithm of Interpretation Power in Criminal Justice Based on Data Activity Consultant
}

\author{
Xiaolong Yang $\mathbb{D}$ \\ Sichuan College of Architectural Technology, Deyang, Sichuan 618000, China \\ Correspondence should be addressed to Xiaolong Yang; 201914070077@zknu.edu.cn
}

Received 16 August 2021; Revised 28 September 2021; Accepted 1 October 2021; Published 15 October 2021

Academic Editor: Fazlullah Khan

Copyright ( $\odot 2021$ Xiaolong Yang. This is an open access article distributed under the Creative Commons Attribution License, which permits unrestricted use, distribution, and reproduction in any medium, provided the original work is properly cited.

In order to solve the problems existing in the current decision algorithm, such as poor data processing performance, low decision accuracy, and long decision time, an automatic decision algorithm of criminal justice interpretation right based on data activity consultant was designed. According to the requirements of reasonable design consultant system data activity and demanded data activity consultants provide data processing requirements and scope. Use the Scrapy web crawler framework to crawl data related to criminal justice interpretation and criminal law provisions from related websites, and clear and extract the collected data to realize data query. Based on the obtained data, the feature array of criminal law is designed, and the decision of criminal judicial interpretation right is made. The C4.5 decision tree algorithm is used to predict the correct rate of decision. The decision of criminal judicial interpretation right is adjusted constantly according to the prediction results to achieve the goal of the automatic decision of criminal judicial interpretation right. Experimental results show that the algorithm has superior data processing performance, high decision accuracy, and short decision time, which verifies the effectiveness of the algorithm.

\section{Introduction}

The personal data activity consultant system was first born in Germany. In accordance with Article 4f, Paragraph 1, of the Federal Data Protection Act [1], public institutions and private law subjects (except private companies with less than 9 persons) automatically collect, process, and use personal data or, otherwise, personal process data and have at least 20 permanent employees. Private subjects that conduct automated data processing for preinspection, transmit or anonymize personal data in business, or automate data processing for market or research purposes must appoint, in writing, a permanent consultant for personal data activities. If a personal data activity adviser is not appointed or does not do so within a prescribed period or in a prescribed manner, or if an appointed personal data activity adviser is unqualified or untrustworthy, the enterprise is liable for an administrative fine of not more than $€ 50,000$.

Data activity consultants should have general knowledge of data protection and the necessary knowledge relevant to the specific data activities of the enterprise involved. Although the criminal law only requires personal data activity consultants to have the necessary skills and does not require specific vocational training, in practice, it is generally required that personal data activity consultants have a "technical background." Personal data activity consultants are highly professional, so they can read the algorithms directly and find problems more easily than ordinary people and administrative officials. The personal data consultants themselves are employees of the enterprise, and the business secrets of the enterprise are protected by the trust relationship between the enterprise and the personal data consultants. The administration has also been appropriately modest, thanks to the presence of consultants on personal data activities. In Germany, personal data activity consultants have always played a major role in regulating corporate data activities. By emphasizing the principle of self-control over data activities, the political system strives to avoid interfering with the enterprise's day-to-day economic activities and maximize respect for corporate freedom. 
Judicial interpretation of criminal law is a kind of authoritative interpretation with the effect of criminal law, and it is the highest level of trial in China. In the specific application of criminal law, procuratorial organs directly clarify the connotation and extension of criminal law norms that procuratorial organs should follow at all levels of trials in the country [2]. Therefore, judicial interpretation of criminal law is of great significance for law enforcement personnel and citizens to correctly understand the exact meaning and scope of application of criminal law norms. It is also one of the important means to materialize and finally realize the legislative intention reflected and embodied by criminal law norms [3]. So this article will discuss data activity consultant where this system is applied to the decision-making of criminal judicial interpretation to make political system prevent the risks of large-scale goals to achieve. Especially given the activities related to the criminal justice and some reasonable explanation and suggestion [4], this would also help ease the pressure of the administrative organ, avoid administration bureaucracy ills, and guarantee social stability [5]. However, most of the current decision algorithms are based on artificial intelligence [6]. However, it is found in the practical application that this algorithm has such problems as poor data processing performance, low decision accuracy, and long decision time, and its practical application effect is not good.

Therefore, this paper mainly carries out the design of automatic decision algorithm of criminal judicial interpretation right with data activity consultant as the core, in order to improve the professionalism and correctness of criminal judicial interpretation right decision and promote the further development of enterprise and society in a safe and stable way.

\section{Design of Automatic Decision Algorithm of Criminal Judicial Interpretation Power}

In the following sections, we provide details about the decision algorithm of criminal judiciary interpretation.

\subsection{Criminal Justice Interpretation Power Data Processing} Based on Data Activity Consultant. In order to improve the accuracy of automatic decision-making of criminal judicial interpretation power, it is necessary to design a reasonable data activity consultant system. Therefore, this paper focuses on the design of the system, and the specific requirements are as follows:

(1) The data activity consultant shall be professional and reliable and meet the qualification requirements specified in the criminal law.

(2) If it fails to perform this obligation, it shall be subject to administrative punishment.

(3) The data activity consultant shall have the obligation of confidentiality and shall not disclose the trade secrets obtained due to his position.

(4) The data activity consultant shall actively impart data activity compliance knowledge to relevant personnel.
(5) The data activity consultant shall participate in the design, inspection, and evaluation of algorithms and write and save algorithm reports.

(6) Data activity consultants shall regularly collect criminal justice information.

(7) The data activity in this paper effectively creates multisystem connection points for an in-depth consultant shall actively cooperate with the criminal law department.

(8) The data activity consultant has the right to seek help and support from the data activity regulatory authority.

(9) If the data activity consultant neglects performing his duties or commits an illegal act jointly with relevant departments, he shall bear joint and several liabilities.

Therefore, through the data activity consultant system, the internal criminal justice organs should be understood to maximize the compliance of data activities without compromising the effectiveness of criminal justice. Today, with the wide application of algorithms and the rapid development of machine learning, data activity consultants can give full play to the role of the whole data collection process of criminal justice interpretation power. Therefore, data activity consultants must understand the data processing program and be notified in time before a new algorithm is implemented. Only in this way can they get enough time to understand and make correct decisions on the power of criminal judicial interpretation. This paper takes the data activity consultant as the core to collect and process the data of the power of criminal judicial interpretation to lay a solid foundation for the follow-up algorithm research. Before collecting and processing data on the right of criminal justice interpretation, design a reasonable data activity consultant system according to relevant requirements, ask the data activity consultant to give relevant data processing requirements and scope, use the Scrapy web crawler framework to crawl criminal law provisions and other data related to the right of criminal justice interpretation from relevant websites, and clean up and extract the original data and realize the data query and display of criminal judicial interpretation power.

The data processing architecture of criminal judicial interpretation power consists of three modules: data acquisition, data processing, and query display. The basic framework of the data processing architecture is shown in Figure 1.

2.1.1. Data Collection Module. All research on criminal law and related contents needs a large number of criminal law contents as support. Therefore, the target data to be collected includes various criminal law provisions. The data acquisition module uses the Scrapy framework and Selenium testing tool to access relevant websites, extract text data in websites, and store it in the MongoDB database for subsequent processing after Bloom filter removal [7]. 


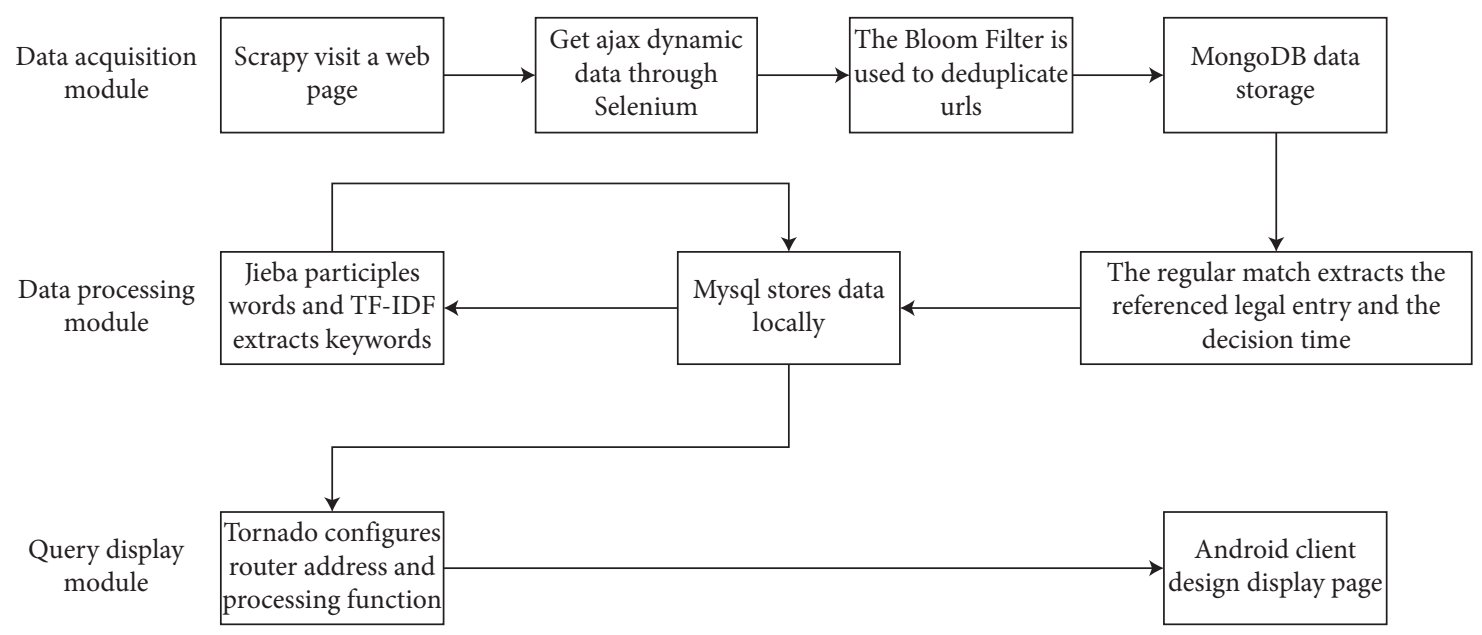

Figure 1: Data processing architecture.

This paper introduces the implementation of Scrapy crawler through the criminal law of a website. First, use Selenium and PhantomJS test tools to access the site's home page, simulate the browser for rendering, and directly grab the rendered page. Then, BeautifulSoup is used to find the navigation tag for the feedback response information, obtain the starting URL address of various criminal provisions, and carry out the deep-first search strategy to crawl the data of criminal provisions [8]. Through observation, it is found that the tag name of each criminal law article is caseName. By obtaining the hyperlink address information in the tag one by one, the URL address of each criminal law article can be obtained by concatenation with the previous category address. At this time, the Bloom filter is used for URL deprocessing, and the target URL is passed into the Bloom filter to check whether the URL exists in the collection [9]. If the URL address already exists in the collection, it will be abandoned; otherwise, it will be queued and the address after hash mapping will be added by 1 [10]. Finally, the URL address is read from the Redis request queue to enter the interface of the body of the criminal law articles. Relevant information such as the title, body content, and execution number of the criminal law articles is obtained. The do-cld attribute value in the request is taken as the unique identifier of the criminal law articles and stored in the MongoDB database [11].

2.1.2. Data Processing Module. As the data obtained by the crawler is rough and unfavorable for subsequent retrieval and analysis, the data processing module is adopted to extract and process the original data collected before and extract the key information from the text. By analyzing the general format of criminal law provisions, appropriate matching template is designed to extract key information and store it in MySQL database. In addition, Jieba word segmentation and Term Frequency-Inverse Document Frequency (TF-IDF) operation are carried out on the contents of criminal law articles to extract the keywords of each criminal law article to facilitate subsequent retrieval and analysis.
In the text keyword extraction part, Jieba word segmentation [12] and TF-IDF technology are adopted. Jieba has three word segmentation modes: precise mode, full mode, and search engine mode. In addition, it can tag the word after word segmentation. Since the precise mode will cut the sentence accurately, which is suitable for text analysis, this paper adopts this mode for word segmentation [13]. In order to improve the accuracy of words, through the research and analysis of massive criminal law provisions, this paper stores customized keywords in Jieba user dictionary and sets the part of speech as $n z$. In the provisions of criminal law, nouns and verbs can better reflect the content of criminal law provisions. Therefore, the parts of speech after text segmentation are extracted from $n$ (noun), $v$ (verb), and $n z$ (custom word) and imported into the bag of words model; the occurrence frequency of keywords is calculated, and the words whose frequency is less than 5 times are eliminated, so as to reduce the influence of incorrect word segmentation and other factors on subsequent processing. Then the TF-IDF operation is carried out on the extracted keywords of criminal provisions. At the same time, in order to improve the accuracy of keyword extraction and overcome the defect that TF-IDF cannot reflect the position information of words, this paper subsections the content of criminal provisions and strengthens the key paragraphs [14].

Tf-IDF is a weight calculation method used to evaluate the importance of words after word segmentation in the text. It is commonly used in fields such as paper mining and keyword extraction [15]. The common formula is as follows:

$$
\mathrm{TF}-\mathrm{IDF}=\frac{N_{W}}{N} \cdot \log \left(\frac{Y}{Y_{W}+1}\right) .
$$

Among them, $N_{W}$ is the number of occurrences of keyword $W$ in criminal law articles, and $N$ is the total number of words after word segmentation in criminal law articles. $Y$ is the total number of criminal law articles, and $Y_{W}$ is the number of criminal law articles including keyword $W$. It is not difficult to see from the formula that although common words appear frequently in the provisions of criminal law, i.e., $N_{W}$ is a large number, there are also many 
provisions of criminal law containing this word, i.e., $Y_{W}$ is also a large number, resulting in the frequency of words in inverse documents being close to 0 and their weight being reduced [16]. Thus, it can be seen that TF-IDF tends to filter out common words and retain important words. After calculating the TF-IDF value of the words in each article of criminal law, the five words with the highest weight value are stored in the database as the keywords representing the criminal law provisions for the convenience of subsequent query [17].

\subsubsection{Querying the Display Module}

(1) Retrieval Display Function Implementation. The data retrieval part is based on the SQLAlchemy framework, which provides an SQL toolkit and object-relational mapping tools. Developers can carry on persistent operation to the database in the way of object oriented, which improves the efficiency of development. The specific use steps are as follows:

(1) Design the required entities in the model file. The entity class inherits the instance of the base class and the field names and attributes are consistent with the corresponding MySQL table structure.

(2) Create MySQL database connection by create_engine () method, associate database table with model file, and set up session dialog.

(3) Import the model file and session dialog into the view file, and then use the query method to operate the MySQL database table under the corresponding routing address. Users can perform multidimensional retrieval operations for data according to different query conditions on the search and query page. The client passes the retrieval field to the appropriate router path address through Okhttp, and SQLAlchemy matches the request field and returns the result to the client. In addition, in order to ensure the user experience and avoid the waste of resources, the Paging module is programmed to perform Paging operations on the data of criminal law provisions and improve the efficiency of system use. When the user rolls the list near the bottom, the background automatically sends a data request to the server to load new data and update the current interface. The request time is short and the interface updates quickly, which improves the robustness of the system. After clicking on the title of a criminal law article, the page will jump to the details page of the criminal law article to view the specific content of the criminal law article. In addition, click the summary button in the upper right corner of the details page to trigger the ToolBar navigation bar, showing the key information of the criminal law article.

(2) Implementation of Recommendation Function. When using the query function, the user may not directly enter the query criteria but need similar results. If there is a recommendation function to provide users with some criminal law provisions related to query conditions, it is very convenient for users.

Recommendation function is based on Word2 $\mathrm{Vec}$, using TF-IDF technology to weight word vector. It strengthens the influence of keywords in criminal law articles, effectively uses word vector to express criminal law articles, calculates the distance of word vector of criminal law articles by cosine similarity formula, and pushes the most similar to the first five criminal law articles. The process is divided into two steps, as follows:

The first step is to generate a vector model of criminal law articles: firstly, the collected criminal law articles are preprocessed by word segmentation to remove symbols and numbers, and relevant part-of-speech words (nouns, verbs, and custom words) are extracted by pos tagging. Then, the training samples were imported into Word2 $\mathrm{Vec}$, and the word vector of each word was obtained by using Skip-Gram model for training. Then, each article of criminal law is divided into words, and the word vector of each word and its TF-IDF word frequency weight value are weighted average, so as to get the document vector and index list of criminal law articles and generate the vector model.

The second step is to calculate the cosine similarity: after the display interface into the criminal law on the page, the provisions of the criminal law will be all documents in the document vector and the vector model vector of the cosine similarity computing, and the similarity of results to sort and take out the top five articles from the index list with related law is recommended to the user interface.

$$
\begin{aligned}
\text { Similarity } & =\cos (\theta)=\frac{A \cdot B}{\|A\|\|B\|} \\
& =\frac{\sum_{i=1}^{n} A_{i} \cdot B_{i}}{\sqrt{\sum_{i=1}^{n}\left(A_{i}\right)^{2}} \cdot \sqrt{\sum_{i=1}^{n}\left(B_{i}\right)^{2}}} .
\end{aligned}
$$

Among them, $A$ and $B$ represent different criminal law article vectors, and $A_{i}$ and $B_{i}$ represent the components of vectors $A$ and $B$.

2.2. Design of Automatic Decision Algorithm of Criminal Judicial Interpretation Power. As for the power of criminal judicial interpretation, it can be divided into several elements of criminal law. An $n$-dimensional array of $\operatorname{Req}[n-1]$ is used as the name of each feature attribute in the appropriate state, and the corresponding value of each element attribute is correspondingly denoted as $V_{\text {Req }[n-1]}$. Under the action of specific application case $k$, where the characteristic of a certain element is $V_{\text {Req }[t]}^{(k)}$, the criminal law characteristic array of criminal judicial interpretation power in general state can be expressed as

$$
R[n-1]^{(k)}=\left\{V_{\text {Req }[0]}^{(k)}, V_{\text {Req }[1]}^{(k)}, \ldots, V_{\text {Req }[t]}^{(k)}, V_{\text {Req }[n-1]}^{(k)}\right\} .
$$


At present, the big data reserve of criminal law cases and criminal law relationship database is obviously insufficient in China, and there is also a lack of corresponding corpus for word segmentation and integration of criminal law language. Therefore, if the intelligent decision-making function by means of machine learning is needed, it can only rely on manual sorting of corresponding database samples. To solve this problem, a training database for a specific class of relationships needs to be established. The field and record structure of the database is shown in Table 1 .

The total number of recorded lines is $D$, the above standard $k$ of each line identifies the specific criminal law situation contained in the data record, the name of the field $\operatorname{Req}[n-1]$ is used to indicate the names of the $n$ elements that may be included in the criminal law relationship, and the Mode field records the observation results of the criminal law relationship model under the case $k$, that is, the cause of action corresponding to the result of historical decision. Different causes correspond to different criminal law relationships. According to the actual situation, $m$ different criminal law relationships can be formed in the database sample, and then the $m$-dimensional array Mode $[m-1]$ can be formed in each record value of field Mode. It should be pointed out that the types of observation results of the criminal law relation model are limited. Although $V_{\text {Mode }}^{(k)}$ represents the value of observation results in line $k$ in the data set, the value of $V_{\text {Mode }}^{(k)}$ will eventually appear in the members of array Mode[m-1]. At the same time, field Jud is formed according to the actual decision results of each recorded case, and the general formula of record values in each field can be seen in Table 1.

In the value of each field, because the characteristic attribute that constitutes a specific criminal law relationship is usually only the dimorphism of "being" and "nothing," the value of field Req $[n-1]$ in each record line can be denoted as " 1 " or "0" correspondingly. Similarly, the value of field Jud is usually 1 or 0 to indicate whether it is correct. As for the value of field Mode, since this field does not need to participate in specific datalization operation, and this field is essentially the observation result of the record row, its value can be any type of data. In order to facilitate the presentation of this paper, in the Mode fields, the continuous natural numbers starting from 1 refer to the relation model of each subclass of criminal law, so as to form a specific array of Mode $[m-1]$, so as to make the preliminary decision on the right of criminal judicial interpretation.

In order to realize the prediction of the correct rate of the decision of the right of interpretation of criminal justice, the first problem to be solved is how to summarize the scattered criminal law data into a specific criminal law relationship model. In this regard, this paper suggests that a complete criminal law relationship database should be established according to the above-mentioned data sample set, and then a certain criminal law data can be classified by using the classification algorithm of data science. Therefore, the core of decision tree classification algorithm is to select the most appropriate feature attribute as the branch node, so that it can predict the classification of each sample more accurately, and the size of the tree is as small as possible, which can
TABLE 1: Field and record structure.

\begin{tabular}{lccccc}
\hline Field name & Req[0] & $\ldots$ & $\operatorname{Req}[n-1]$ & Mode & Jud \\
\hline First line values & $V_{\text {Req[0] }}^{(1)}$ & $\ldots$ & $V_{\text {Req }[n-1]}^{(1)}$ & $V_{\text {Mode }}^{(1)}$ & $V_{\text {Jud }}^{(1)}$ \\
The $k$-th row takes value & $V_{\text {Req[0] }}^{(k)}$ & $\ldots$ & $V_{\text {Req[n-1] }}^{(k)}$ & $V_{\text {Mode }}^{(k)}$ & $V_{\text {Jud }}^{(k)}$ \\
$\begin{array}{l}\text { At the end of the row } \\
\text { values }\end{array}$ & $V_{\text {Req[0] }}^{(D)}$ & $\ldots$ & $V_{\text {Req }[n-1]}^{(D)}$ & $V_{\text {Mode }}^{(D)}$ & $V_{\text {Jud }}^{(D)}$ \\
\hline
\end{tabular}

establish a reasonable connection with the goal of the decision of criminal judicial interpretation right and become the most appropriate classification algorithm. The comparison of three different mainstream decision trees is shown in Table 2.

In this paper, referring to the classical algorithm of C4.5, the classification of criminal law situation is appropriately improved. The following three steps are used to realize the classification of criminal law relationship model.

(1) Merge the record rows with the same feature value Because the decision-making of the power of interpretation of criminal justice pursues the correctness to the greatest extent, the model base of criminal law relations for learning and training should be as comprehensive and concise as possible. Under the requirements of this demand, the training sample set shown in Table 1 has obvious deficiencies. On this basis, the data of criminal judicial interpretation power should be integrated and optimized. In order to achieve accurate classification by decision tree, only the most accurate observation result records should be retained in the criminal law relationship database. In short, it is to merge all different record lines with identical feature values and retain only one record line for different feature value records. In fact, field Jud of decision results has no connection with the establishment of the criminal law relational model, so this field can be directly deleted or ignored in the criminal law relational model base. Thus, the most comprehensive sample base of the probability of occurrence of various criminal law relation models can be formed in the theoretical state.

In addition, due to the consideration of the comprehensibility of the criminal law relation model in the database, the missing record lines can be artificially supplemented to form all possible fact records in the ideal state and make the criminal law relation model database complete.

$$
\begin{aligned}
H(\operatorname{Req}[t])= & -\sum_{i=0}^{\max \left(V_{\text {Req }[t]}\right)} \frac{D\left(V_{\text {Req }[t]}=i\right)}{D} \\
& \cdot \log _{2} \frac{D\left(V_{\text {Req }[t]}=i\right)}{D} .
\end{aligned}
$$

After the combined record lines, the split information entropy of the feature attributes of each constitutive element can be calculated according to the 
TABLE 2: Characteristics comparison of three types of typical decision tree algorithms.

\begin{tabular}{|c|c|c|c|c|c|}
\hline The algorithm name & Support model & Tree structure & $\begin{array}{c}\text { Identify } \\
\text { characteristics }\end{array}$ & Eigenvalue type & Tree pruning \\
\hline ID3 & Classification & Many tree & Information gain & Enumerated values & Does not support \\
\hline $\mathrm{C} 4.5$ & Classification & Many tree & Information gain rate & $\begin{array}{l}\text { Enumeration value, } \\
\text { continuous value }\end{array}$ & Support \\
\hline CART & Classification and regression & Binary tree & The Gini coefficient & $\begin{array}{l}\text { Enumeration value, } \\
\text { continuous value }\end{array}$ & Support \\
\hline
\end{tabular}

above equation, where $D$ represents the number of record lines that meet the specific conditions in the combined criminal law relation model base. The calculated split information entropy of good $H(\operatorname{Req}[t])$ can intuitively show the complexity of a certain characteristic attribute. The higher the value of entropy is, the more unstable the corresponding constitutive requirements of criminal law in the ideal state will be, and the more feasible it will be to change to other similar constitutive requirements.

(2) Compute the information gain of characteristic values

Information gain is another metric for data attributes that is different from information entropy in the basic theory of data science. The more important the influence of an attribute on the observation results is, the more the uncertainty space for classification will be eliminated by it, that is, the more the information for classification will be provided. From the perspective of the fuzziness of criminal law relations, if a certain constitutive element Req $[t]$ is more important to the determination of criminal law relations, the form of its corresponding element attribute in the data sample set is less complex, that is, the information gain is greater, and it is easier to determine it in the decision-making process of criminal judicial interpretation power. Therefore, the following formula for calculating the gain of component element $G=$ (Mode, $\operatorname{Req}[t])$ is proposed in this paper, which is analogous to the calculation method of information gain in data science

$G($ Mode, Req $[t])=H($ Mode $)-H($ Mode, Req $[t])$

$$
\begin{aligned}
= & \sum_{i=0}^{\max \left(V_{\mathrm{Req}[t]}\right)} \frac{D\left(V_{\mathrm{Req}[t]}=i\right)}{D} \cdot\left(\sum_{j=0}^{m-1} \frac{D\left(V_{\mathrm{Req}[t]}=i \mid \operatorname{Mode}[j]\right)}{D\left(V_{\operatorname{Req}[t]}=i\right)} \cdot \log _{2} \frac{D\left(V_{\mathrm{Req}[t]}=i \mid \operatorname{Mode}[j]\right)}{D\left(V_{\mathrm{Req}[t]}=i\right)}\right) \\
& -\sum_{i=0}^{n-1} \frac{D\left(V_{\operatorname{Req}[t]}=i\right)}{D} \cdot \log _{2} \frac{D(\operatorname{Mode}[i])}{D} .
\end{aligned}
$$

$H$ (Mode) represents the category information entropy of the observed results of the criminal law relationship model in the data sample, and $H$ (Mode|Req $[t]$ ) refers to the information entropy of the specific attribute Req[t]. In terms of data results, the corresponding information gain of constitutive elements can reflect the certainty and accuracy of the major premise and minor premise in the process of criminal reasoning.

(3) Recursively constructed tree model

After the information splitting entropy and information gain of a certain constitutive element are calculated in the first two steps, the information gain rate of feature $G_{R}[t]$ of this constitutive element in a specific sample data set can be obtained according to the conceptual definition of information gain rate in information science according to the formula below, and a set $G_{R}[n-1]$ composed of information gain rates of all feature attributes can be formed.

$$
G_{R}[t]=\frac{G(\text { Mode, } \operatorname{Req}[t])}{H(\operatorname{Req}[t])} \text {. }
$$

According to the above form of information gain rate collection, select one of the biggest values of $\operatorname{Max}\left(G_{R}[n-1]\right)$ as labels for a particular data set for the first time classification properties; thus the binary or fork forms a number of data sets, again on the basis of the above three steps of the recursive classification, screening each subsample of the maximum of information gain rate points to the attributes. Use this as a tag attribute for subclassing. Finally, the smallest subclass obtained by recursion has the same observation result of the criminal law relation model; that is, it 
conforms to the algorithm objective of constructing the tree to determine the classification standard, and the label of each branch node is clear. Therefore, the construction of criminal law classification decision tree is completed by learning and training according to the existing data samples, and then the unknown results of the criminal justice interpretation right facts can be included or classified forecast according to this tree model.

Naive Bayes is a classification method based on the mathematical Bayes' theorem and the independent assumption of characteristic attributes on the corresponding data. The data conclusions obtained by naive Bayes algorithm are generally presented in the form of probability. In the context of criminal law application, naive Bayesian algorithm can be used to predict or analyze the risk of the correct rate of criminal judicial interpretation right decision based on historical data. The realization process is to learn and train the data samples and conclude the specific probability model according to the rules embodied in the samples. To realize the prediction of the probability conclusion of specific unknown facts according to the model and application model, based on the data table structure model established in Table 1, the improved application of naive Bayes algorithm in criminal law scenes and the realization of the corresponding automatic decision algorithm of criminal judicial interpretation right are explained as follows.

Under the naive Bayes algorithm of probability model is actually according to the observations in the training sample prior probability forecast a posteriori probability but needs to assume that the prior probability calculation process conditions are independent of each other and do not have relationship between the cross, but the premise is basic scenario of criminal law that can be in accordance with it; the constitutive requirements of a scientific theory of the basic criminal law are independent elements. Therefore, under this premise, the calculation formula of prior probability can be deduced from the combination of known facts feature elements $R[n-1]^{(k)}$ for the unknown disputes that conform to all the features of constitutive elements in line $k$ under the classification of inclusion and absorption, where $P(\mathrm{Jud}=1)$ represents the accuracy of observation results recorded by all data, and $R(\operatorname{Req}[n-1])=R[n-1]^{(k)} \mid \mathrm{Jud}=1$ represents the corresponding accuracy of each feature under the condition of matching the value of each feature in the feature set of facts.

$$
\begin{aligned}
P(\mathrm{Jud}=1) & =\frac{D\left(V_{\mathrm{Jud}}=1\right)}{D}, \\
R\left(\operatorname{Req}[n-1]=R[n-1]^{(k)} \mid \mathrm{Jud}=1\right) & =\prod_{i=0}^{n-1} P\left(\operatorname{Req}[i]=R[i]^{(k)} \mid \mathrm{Jud}=1\right) \\
& =\prod_{i=0}^{n-1} \frac{D\left(V_{\mathrm{Req}[i]}=R[i]^{(k)} \mid V_{\mathrm{Jud}}=1\right)}{D\left(V_{\mathrm{Jud}}=1\right)} .
\end{aligned}
$$

In addition, it is also necessary to calculate the total probability $R\left(\operatorname{Req}[n-1]=R[n-1]^{(k)}\right)$ for the value of each feature in the factual feature set, that is, the sum of the correct rate and error rate for the value of each conditional feature. The calculation formula is as follows:

$$
\begin{aligned}
P\left(\operatorname{Req}[n-1]=R[n-1]^{(k)}\right) & =\sum_{i=0}^{\max \left(V_{\text {Jud }}\right)}\left(P(\mathrm{Jud}=i) \cdot P\left(\operatorname{Req}[n-1]=R[n-1]^{(k)} \mid \mathrm{Jud}=i\right)\right) \\
& =\frac{D\left(V_{\text {Jud }}=1\right)}{D} \cdot \prod_{i=0}^{n-1} \frac{D\left(V_{\mathrm{Req}[i]}=R[i]^{(k)} \mid V_{\text {Jud }}=1\right)}{D\left(V_{\text {Jud }}=1\right)}+\frac{D\left(V_{\text {Jud }}=0\right)}{D} \cdot \prod_{i=0}^{n-1} \frac{D\left(V_{\text {Req }[i]}=R[i]{ }^{(k)} \mid V_{\text {Jud }}=0\right)}{D\left(V_{\text {Jud }}=0\right)} .
\end{aligned}
$$

Thus according to Bayes' theorem, from the results of the prior probability and the probability to deduce specific criminal law relations under the model of criminal judicial interpretation accuracy probability model, the probability model of the practical significance is the historical decision results and the unknown criminal judicial interpretation 
TABLE 3: Experimental environment.

\begin{tabular}{lcc}
\hline Experimental environment & Configuration & Parameter \\
\hline & CPU & Intel (R) Core (TM) i5-9400 \\
Hardware environment & Frequency & $2.90 \mathrm{GHz}$ \\
& RAM & $16.0 \mathrm{~GB}$ \\
\hline & The operating system & Windows 10 \\
Software environment & Version & 18362.1082 pro \\
& Digits & 64 bit \\
& Analog software language & APDL \\
& The simulation software & Matlab 7.0 \\
\hline
\end{tabular}

decision-making process; the results established a degree of connection, making the former to the latter provides a reasonable prediction.

$$
P\left(\operatorname{Jud}=1 \mid \operatorname{Req}[n-1]=R[n-1]^{(k)}\right)=\frac{P(\operatorname{Jud}=1) \cdot P(\operatorname{Req}[n-1])=R[n-1]^{(k)} \mid \mathrm{Jud}=1}{P\left(\operatorname{Req}[n-1]=R[n-1]^{(k)}\right)}
$$

As shown in the above equation, the posterior probability obtained from the combination of certain characteristic elements by this equation is the correct rate of predicting the decision of criminal judicial interpretation power according to the constitutive elements of criminal law relations formed by it. When the probability of correct rate is higher than 0.9 , it is generally considered that the decision of criminal judicial interpretation power is high and the result can be directly output. However, if the probability is lower than 0.9 , it is necessary to constantly adjust the decision result, so as to achieve the goal of improving the correct rate of automatic decision of criminal judicial interpretation power.

\section{Experimental Verification and Result Analysis}

3.1. Experimental Design. In order to verify the effectiveness of the automatic decision algorithm of criminal judicial interpretation power based on data activity consultant, an experiment was designed. The specific experimental environment is shown in Table 3.

Due to the complexity of criminal law relationship, the number of constituent elements is relatively large. This paper takes a simple criminal law relationship with 7 elements as an example and puts all of it into the data sample and participates in the algorithm implementation. On this basis, all judicial interpretation information related to the provisions of the criminal law will be collected, and the collected data will be cleaned and processed, which will be taken as experimental sample data, so as to verify the judgment algorithm proposed in this paper.

3.2. Analysis of Experimental Results. In order to verify the effectiveness of the designed automatic decision algorithm of criminal judicial interpretation power with data activity consultant as the core, the time of data collection, processing, and query under different data levels is first tested. The specific results are shown in Table 4.

With the increase of amount of experimental data, this paper's algorithm of data acquisition, processing, and query time tends to rise, and under the condition of the experimental data of $500 \mathrm{~GB}$, the algorithm of data acquisition, processing, and query time reached the maximum value, a maximum time of $1.37 \mathrm{~s}$ of data collection, and data processing time to a maximum of $1.42 \mathrm{~s}$. The data query time is $0.61 \mathrm{~s}$, and the data collection, processing, and query time of this algorithm are low, which meets the research expectations. On the above basis, the decision accuracy of the algorithm in this paper is compared with the automatic decision algorithm based on artificial intelligence. The decision accuracy refers to the degree of similarity between the decision results of different methods and the decision results of authorities. The specific comparison results are shown in Table 5 .

By analyzing the data in Table 5, we can see that the accuracy of automatic decision-making of criminal judicial interpretation power of this algorithm is $96.9 \%$ at the highest and $93.8 \%$ at the lowest. The accuracy of automatic decisionmaking of criminal judicial interpretation power based on artificial intelligence algorithm is $86.5 \%$ at the highest and $71.9 \%$ at the lowest. Compared with the automatic decisionmaking algorithm based on artificial intelligence, the automatic decision-making accuracy of criminal judicial interpretation power of this algorithm is higher, which can lay a solid foundation for the analysis and research of criminal judicial interpretation power.

Finally, the decision-making time of this algorithm and the automatic decision-making algorithm based on artificial intelligence is compared, and the comparison results are shown in Figure 2.

By analyzing the data in Figure 2, it can be seen that the automatic decision-making time of criminal judicial 
TABLE 4: Experimental test of data collection, processing, and query time.

\begin{tabular}{lccc}
\hline Data volume $(\mathrm{GB})$ & Data collection time $(\mathrm{s})$ & Data processing time $(\mathrm{s})$ & Data query time $(\mathrm{s})$ \\
\hline 50 & 0.23 & 0.25 & 0.35 \\
100 & 0.34 & 0.33 & 0.36 \\
150 & 0.56 & 0.48 & 0.42 \\
200 & 0.68 & 0.57 & 0.46 \\
250 & 0.79 & 0.69 & 0.48 \\
300 & 0.95 & 0.73 & 0.51 \\
350 & 1.21 & 0.88 & 0.54 \\
400 & 1.36 & 0.91 & 0.56 \\
450 & 1.58 & 1.31 & 0.58 \\
500 & 1.37 & 1.42 & 0.61 \\
\hline
\end{tabular}

TABle 5: Comparison of decision accuracy (\%).

\begin{tabular}{lcc}
\hline Data volume $(\mathrm{GB})$ & The algorithm designed in this paper & Automatic decision algorithm based on artificial intelligence \\
\hline 50 & 95.3 & 86.5 \\
100 & 95.1 & 84.7 \\
150 & 96.9 & 82.5 \\
200 & 94.2 & 81.3 \\
250 & 93.8 & 79.6 \\
300 & 94.7 & 74.5 \\
350 & 93.8 & 72.6 \\
400 & 94.7 & 74.6 \\
450 & 95.8 & 72.8 \\
500 & 96.3 & 71.9 \\
\hline
\end{tabular}

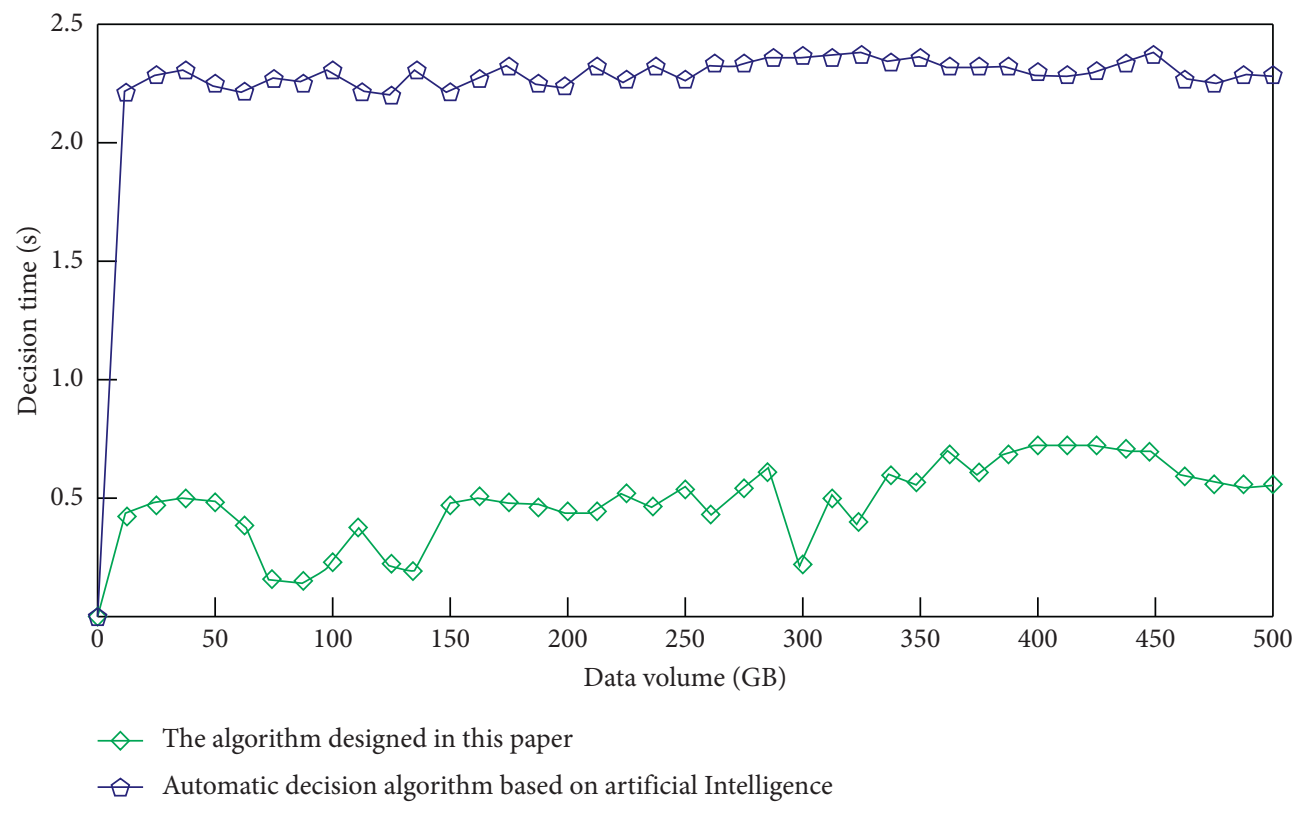

Figure 2: Comparison of decision time.

interpretation power of the algorithm in this paper varies from $0 \mathrm{~s}$ to $0.65 \mathrm{~s}$, and the automatic decision-making time of criminal judicial interpretation power of the automatic decision-making algorithm based on artificial intelligence varies from $0 \mathrm{~s}$ to $2.39 \mathrm{~s}$. Compared with the automatic decision-making algorithm based on artificial intelligence, the automatic decision-making time of criminal judicial interpretation power of the algorithm in this paper is shorter. The overall operation efficiency is higher, and the automatic decision-making of criminal judicial interpretation power can be realized faster.

\section{Conclusion}

The automatic decision algorithm of criminal judicial interpretation power is not only a criminal law problem, but 
also a political and economic problem. The political system needs to prevent social unrest caused by large-scale harm. Therefore, it is of great significance to design the automatic decision-making algorithm of criminal judicial interpretation power. Therefore, in order to solve the problems existing in the current algorithm, such as poor data processing performance, low decision accuracy, and long decision-making time, this paper designs an automatic decision-making algorithm of criminal judicial interpretation power with data activity consultant as the core. According to the requirements, the data activity consultant gives the relevant data processing requirements and scope, collects the criminal law provisions and other data by using the Scrapy web crawler framework, processes the collected data, and provides the data query and display function, so as to complete the design of the data processing architecture. On this basis, the array of criminal law characteristics is designed, and the decision of criminal judicial interpretation power is made. C4.5 decision tree algorithm is used to predict the accuracy of decision-making, and the decisionmaking of criminal judicial interpretation power is continuously adjusted according to the prediction results, so as to achieve the goal of automatic decision-making of criminal judicial interpretation power. The experimental results show that the maximum data acquisition time of this algorithm is $1.37 \mathrm{~s}$, the maximum data processing time is $1.42 \mathrm{~s}$, and the data query time is $0.61 \mathrm{~s}$. The data acquisition, processing, and query time are low. The highest accuracy of automatic decision-making of criminal judicial interpretation power is $96.9 \%$, the lowest is $93.8 \%$, and the decision-making time varies from $0 \mathrm{~s}$ to $0.65 \mathrm{~s}$. It can realize the correct and rapid decision-making of the power of criminal judicial interpretation and can provide important theoretical reference for relevant research.

\section{Data Availability}

The data used to support the findings of this study are available from the corresponding author upon request.

\section{Conflicts of Interest}

The authors declare that they have no known competing financial interests or personal relationships that could have appeared to influence the work reported in this paper.

\section{References}

[1] L. Kong, "Enacting China's data protection act," International Journal of Law and Information Technology, vol. 18, no. 3, pp. 197-226, 2010.

[2] P. Wang, "Thoughts on the right of interpretation of criminal law-on the right of interpretation of criminal law and judicial interpretation," Journal of Hebei Normal University of Science and Technology, vol. 18, no. 2, pp. 46-50, 2019.

[3] Q. $\mathrm{Xu}$, "Research on the time validity of criminal law interpretation," Theory Horizon, vol. 52, no. 1, pp. 57-63, 2019.

[4] S. Walker, "R v BM: errors in the judicial interpretation of body modification," Journal of Criminal Law, vol. 83, no. 4, pp. 245-257, 2019.
[5] M. Zalnieriute and C. Weiss, "Reconceptualizing intersectionality in judicial interpretation: moving beyond formalistic accounts of discrimination on islamic covering prohibitions," Berkeley Women's Law Journal, vol. 35, no. 1, pp. 71-90, 2020.

[6] E. D. Zhang, "Challenge of artificial intelligence algorithm decision-making to administrative rule of law and institutional response," Research on Administrative Law, vol. 56, no. 4, pp. 34-45, 2020.

[7] B. Jerman and T. Klobuar, "A new legal framework for crossborder data collection in crime investigation amongst selected european countries," International Journal of Cyber Criminology, vol. 13, no. 2, pp. 1-12, 2019.

[8] E. B. Esien, "Jozefien van Caeneghem. 2019. Legal Aspects of Ethnic data collection and positive action: the roma minority in Europe. Cham: Springer nature Switzerland. 728 pp," Nordic Journal of Migration Research, vol. 11, no. 2, pp. 228-231, 2021.

[9] L. Luo, D. Guo, R. T. Ma, O. Rottenstreich, and X. Luo, "Optimizing bloom filter: challenges, solutions, and comparisons," IEEE Communications Surveys and Tutorials, vol. 21, pp. 1912-1949, 2018.

[10] S. X. Wang, Y. Z. Chen, and S. T. Zhang, "Acquisition and application of social observation data based on reptile technology," Progress of Meteorological Science and Technology, vol. 9, no. 3, pp. 234-237, 2019.

[11] Z. X. Zeng, C. D. Zou, and J. F. Wei, "optimization of distribution cost model of cold chain logistics for litchi based on ant colony algorithm," Packaging Engineering, vol. 40, no. 11, pp. 58-65, 2019.

[12] Q. B. Wang, Q. Q. Chen, and L. B. Wang, "Design of one-stop service system for medical equipment information query based on Jieba participle," China Medical Equipment, vol. 17, no. 1, pp. 138-141, 2020.

[13] F. G. Shi, "Implementation of Chinese text corpus preprocessing module based on Jieba Chinese word segmentation," Computer Knowledge and Technology, vol. 16, no. 14, pp. 254-257, 2020.

[14] Z. L. Zhang and P. Z. Yang, "Large scale text preprocessing based on self-encoder semantic hashing," Computer Simulation, vol. 36, no. 3, pp. 231-235, 2019.

[15] H. E. Saihanqiqige, "Application research of English scoring based on TF-IDF clustering algorithm," IOP Conference Series: Materials Science and Engineering, vol. 750, no. 1, pp. 12215-12301, 2020.

[16] Z. Jiang, B. Gao, Y. He, Y. Han, P. Doyle, and Q. Zhu, “Text classification using novel term weighting scheme-based improved TF-IDF for Internet media reports," Mathematical Problems in Engineering, vol. 2021, no. 6, 30 pages, Article ID 6619088, 2021.

[17] L. Zhang, "Research on case reasoning method based on TFIDF," International Journal of System Assurance Engineering and Management, vol. 12, no. 3, pp. 608-615, 2021. 